\title{
Mejora en algunas dimensiones de salud percibida en pacien- tes con fibromialgia mediante la aplicación de un programa de meditación mindfulness
}

\author{
Clemente Franco Justo, Israel Mañas Mañas y Eduardo Justo Martínez \\ Universidad de Almería. \\ (Recibido 25 de Mayo 2010; Aceptado 27 Septiembre 2010)
}

\begin{abstract}
RESUMEN: En este estudio se analizan los efectos que un programa de meditación para el desarrollo de la conciencia plena (mindfulness), puede tener sobre la salud percibida en un grupo de pacientes diagnosticados con fibromialgia. Participaron 33 pacientes diagnosticados de fibromialgia. Se realizó un diseño cuasiexperimental con medición pretest-postest-seguimiento, con un grupo experimental y un grupo control. Como instrumento de evaluación se utilizó el Cuestionario SF-36. Los análisis estadísticos realizados muestran una mejora en las dimensiones funcionamiento físico, dolor corporal, salud general, funcionamiento social, rol emocional, salud mental, y componente mental una vez finalizada la intervención. Estas mejoras se mantienen en la evaluación de seguimiento realizada tres meses después de finalizada la intervención.

Estos resultados son acordes con otras investigaciones donde se ha comprobado la eficacia de las técnicas de mindfulness en la mejora de pacientes con fibromialgia. No obstante, el estudio presenta varias limitaciones que habría que subsanar en sucesivas investigaciones para dotar de mayor validez a los resultados obtenidos.
\end{abstract}

Palabras clave: meditación, conciencia plena, fibromialgia, salud percibida, intervención.

\section{Perceived health improvement through a mindfulness meditation development} program in fibromyalgia patients

ABSTRACT: This study analyzes the effects of a mindfulness development meditation program on perceived health in a group of patients diagnosed with fibromyalgia. Thirty-three patients diagnosed with fibromyalgia participated. A quasi-experimental pretest/posttest/follow-up design was carried out, with one experimental group and one control group. The SF-36 Questionnaire was used as the evaluation instrument.

The statistical analyses done show an improvement in the dimensions physical functioning, bodily pain, general health, social functioning, role emotional, mental health, and mental component at the end of the intervention. These improvements are maintained in the follow-up test given three months after intervention. These results are in agreement with other studies which have found mindfulness techniques to be effective in improving patients with fibromyalgia. Nevertheless, the study shows various limitations that would have to be corrected in successive studies to bring more validity to the results.

Keywords: meditation, mindfulness, fibromyalgia, perceived health, intervention 
La fibromialgia es una patología de naturaleza crónica que afecta a nivel biológico (falta de energía, dolor, etc.), psicológico (ansiedad, depresión, etc.), y social, y que afecta a la calidad de vida tanto a nivel personal, social como laboral (Collado et al., 2002).

Muchos de estos pacientes muestran alteraciones comportamentales, a la vez que muestran una vivencia y un enfrentamiento deteriorado al dolor que padecen, por lo que la fibromialgia encaja perfectamente dentro de los trastornos psicosomáticos del área afectiva (Castro, 2007). En este sentido, Garofalo (2000) establece que las variables psicológicas están implicadas de forma importante en la experiencia de dolor, al tiempo que presentan un papel fundamental en la respuesta que los pacientes presentan al tratamiento. Así, Turk (1996) establece que el desarrollo y manifestación del dolor y la fatiga características de la fibromialgia, están originadas por un proceso de condicionamiento respondiente. De este modo, un paciente con vulnerabilidad psicológica, perpetúa los síntomas de dolor y de fatiga mediante la exposición repetida a ciertos estímulos.

La elevada prevalencia que hoy día está alcanzando el síndrome de fibromialgia, y las graves repercusiones sociales, familiares, laborales, económicas y sanitarias que conlleva, hacen necesaria la búsqueda constante de tratamientos que puedan paliar y mejorar los síntomas de esta enfermedad hasta hoy día incurable (Carricondo, 2009) .

Es por ello, que las técnicas de meditación mindfulness para el desarrollo de la conciencia plena, suponen hoy día en el campo de la medicina conductual y de la psicología, un adecuado recurso terapéutico para paliar, prevenir y contrarrestar muchos de los síntomas manifestados por los pacientes con fibromialgia, dado su carácter grupal y psicoeducativo, y porque son varios los estudios que han comprobado una mejora en el dolor, en la calidad de vida, en síntomas somáticos, en ansiedad y en depresión en pacientes con fibromialgia mediante el empleo de este tipo de técnicas (Grossman, Tiefenthaler-Gilmer, Raysz y Kiespes, 2007; Sephton et al., 2007) .

El objetivo de las técnicas de mindfulness es que los participantes tengan experiencias de conciencia plena, en el sentido de "dejarse llevar" por las sensaciones que perciben en cada momento. Es decir, se trata de promover, de poner como punto fundamental de referencia, las sensaciones y emociones, dejando que ellas actúen de forma natural. Esto posibilita que la experiencia que en ese momento tiene la persona fluya, permitiendo que determinadas actividades (emociones, cambios fisiológicos, etc.) que operan de forma autónoma en el organismo, regulados por el sistema nervioso autónomo, se regulen de acuerdo con sus propios sistemas naturales de autorregulación (Vallejo, 2006).

Por tanto, el supuesto que subyace a las técnicas de meditación mindfulness es que la falta de información sensorial, ya sea activa (mediante el uso de técnicas de control, distracción, etc.), o bien pasiva (no atendiendo de forma intencional a dicha información), impiden al organismo la información precisa y necesaria para que tengan lugar los modos naturales de aprendizaje (Vallejo, 2007). Este planteamiento, encajaría perfectamente con diversos estudios que han señalado 
como las estrategias de afrontamiento pasivas (como el pensamiento catastrofista) incrementan la percepción subjetiva del dolor y el malestar psicológico, mientras que la utilización de estrategias de afrontamiento activas están relacionadas con un mejor estado de ánimo y un mejor grado de funcionamiento (Brown, Nicasio y Wallston, 1989; Soriano y Monsalve, 1999).

Dado que la calidad de vida relacionada con la salud, se ha convertido en los últimos años en un indicador de la efectividad de una intervención (Durán, Gallegos, Salinas y Martínez, 2004), el objetivo de la presente investigación fue comprobar los efectos de un programa psicoeducativo de mindfulness aplicado en formato grupal, sobre la salud percibida de un grupo de pacientes diagnosticados con fibromialgia.

\section{MÉTODO}

\section{Diseño}

Para analizar los efectos del programa de meditación mindfulness (variable independiente) sobre las dimensiones de salud percibida de los pacientes con fibromialgia (variables dependientes), se utilizó un diseño de tipo cuasiexperimental con medición pretest-postest-seguimiento, con un grupo experimental y un grupo control en espera.

\section{Participantes}

La muestra del presente estudio estuvo constituida por 33 mujeres diagnosticadas de fibromialgia pertenecientes a la Asociación de Fibromialgia de Almería (AFIAL), cuyas edades oscilaban entre los 39 y los 66 años $(\mathrm{M}=56.87$; $\mathrm{DT}=11.69)$.

\section{Instrumentos}

Para la evaluación de la salud percibida de las pacientes con fibromialgia se utilizó el cuestionario SF-36 (Alonso, Prieto y Antón, 1995), que es un instrumento genérico de medida en el que la persona valora su estado de salud según la percibe, y que contempla aspectos tanto de salud física, como psíquica y social (García, Santibáñez y Soriano, 2004). Consta de 36 ítems distribuidos en 8 dimensiones o escalas de salud percibida, más una evaluación del estado de salud actual comparado con hace un año que se evalúa con un solo ítem. Además algunos estudios (McHorney, Ware y Raczeck, 1993; Vilagut, Valderas, Ferrer, Garin, López-García y Alonso, 2008) han confirmado la existencia de dos componentes principales de salud, el dominio físico y el mental, cuyos coeficientes de consistencia interna están próximos a 0.90 (Alonso, Regidor, Barrio, Prieto, Rodríguez y de la Fuente, 1998).

En cada ítem la puntuación obtenida se transforma en una escala de $0-100$ 
según el porcentaje alcanzado de la máxima puntuación posible, indicando los valore mayores una mejor calidad de vida.

Las puntuaciones de las dimensiones del SF-36 no pueden ser interpretadas en sí mismas, sino que deben ser transformadas en una escala de significación uniforme y común a todas las dimensiones, mediante la comparación con las puntuaciones obtenidas en el subgrupo de las muestras normativas en función del género y el grupo de edad (Alonso et al., 1998).

En su versión original (Ware y Sherbourne, 1992) el SF-36 posee una elevada consistencia interna con un coeficiente alpha de Cronbach de 0.80 para todas las escalas excepto para la de función social que es de 0.76 . Los valores psicométricos de la versión española son parecidos a los de la versión original, excepto en lo que respecta a la fiabilidad de la dimensión función social que es un poco menor (Ayuso-Mateos, Lasa y Vázquez-Barquero, 1999).

\section{Procedimiento}

En primer lugar, se contactó con la asociación AFIAL para ofrecerles un curso titulado "Entrenamiento y práctica de la conciencia plena en pacientes con fibromialgia" al cual se inscribieron un total de 39 personas, de las cuales 20 formaron parte del grupo experimental, y las 19 restantes formaron parte del grupo control. La asignación a uno u otro grupo se realizó de manera aleatoria al azar. De las 20 personas que formaron parte del grupo experimental, 5 de ellas no finalizaron el curso o no cumplieron con los requisitos para formar parte de la investigación, por lo que la muestra final de este grupo estuvo constituida por 15 personas, quedando reducida la muestra final de la investigación a 33 participantes, ya que un sujeto del grupo control no realizó la evaluación de seguimiento. A los sujetos del grupo control, se les informó que por razones de espacio ellos recibirían el curso en un segundo turno.

Antes del comienzo del curso, se procedió a obtener la medida pretest de los niveles de salud percibida, para lo cual se facilitó a todas las participantes inscritas en el curso el cuestionario SF-36 para que lo cumplimentasen de forma individual.

A continuación, se procedió a la aplicación del programa de intervención en el grupo experimental, realizándose una sesión semanal de dos horas de duración cada una de ellas, consistente en un programa psicoeducativo de meditación mindfulness para el desarrollo de la conciencia plena (Franco, 2009). Este programa es una adaptación del programa de reducción del estrés denominado Mindfulness-Based Stress Reduction (MBSR) (Kabat-Zinn, 1990), con la introducción de estrategias mindfulness utilizadas en la terapia de conducta dialéctica (Linehan, 1993) y en la terapia de aceptación y compromiso (Hayes, Strosahl y Wilson, 1999; Wilson y Luciano, 2002).

Durante la realización de las diferentes sesiones, se persigue la consecución de tres objetivos principales: 
1. Tomar conciencia de la tendencia de la mente a oscilar entre el pasado y el futuro, al tiempo que se resiste a permanecer en el momento presente, en el aquí y ahora.

2. Ser conscientes de que los pensamientos, sensaciones y emociones, están surgiendo y desapareciendo continuamente, están fluyendo de forma constante, por lo que todos ellos comparten la misma naturaleza y es que surgen y desaparecen a cada instante, por lo que es importante aprender a observar su carácter transitorio y no permanente.

3. Aprender a observar como consumimos mucha energía diariamente tratando de evitar una serie de pensamientos, sentimientos, emociones y sensaciones que consideramos molestos y negativos, en lugar de dejarlos fluir libremente y concentrarnos en realizar aquellas tareas o actividades importantes que debemos de resolver en cada momento.

El programa fue desarrollado por un instructor con una amplia experiencia tanto en la práctica como en la enseñanza de técnicas de meditación mindfulness, y las diferentes sesiones del programa de intervención tuvieron la siguiente estructura:

1. Comentarios de los participantes sobre su práctica de los ejercicios de meditación mindfulness durante la semana (dudas, experiencias, contratiempos).

2. Realización del ejercicio de recorrido corporal durante 10 minutos.

3. Presentación de las diversas metáforas y ejercicios correspondientes a cada sesión.

4. Práctica de conciencia plena atendiendo a la respiración durante $30 \mathrm{mi}$ nutos.

Para facilitar la implementación del programa, los sujetos tenían que realizar diariamente en casa el ejercicio de recorrido corporal durante 10 minutos, y la práctica de conciencia plena sobre la respiración en la zona del abdomen durante 30 minutos. Además, cada participante debía completar a lo largo de la semana una hoja de autorregistro en la que diariamente debía anotar si había practicado los ejercicios de recorrido corporal y de conciencia plena en la respiración mandados para realizar en casa. El grado medio de cumplimiento del ejercicio de recorrido corporal fue del $64 \%$, mientras que del ejercicio de conciencia plena fue del $79 \%$. No se tuvieron en cuenta para los resultados del estudio, a aquellos participantes que acudieron a menos del $50 \%$ de las sesiones del programa de intervención, y que en la hoja de autorregistro informaron de haber practicado menos del $50 \%$ de los días el ejercicio de recorrido corporal y/o el ejercicio de conciencia plena.

Una vez finalizado el programa de intervención se procedió a obtener las medidas postest, para lo cual se volvió a administrar a todas las participantes el cuestionario SF-36 con el fin de comprobar si se habían producido modificaciones significativas en sus niveles de salud percibida. 
Con el objetivo de comprobar si los resultados obtenidos en los niveles de salud percibida se mantenían a lo largo del tiempo, tres meses después de finalizada la intervención se realizó una medida de seguimiento siguiéndose el mismo procedimiento de medición que en las fases pretest y postest.

Una vez finalizada la evaluación de seguimiento, se procedió a impartir el curso de meditación mindfulness a los sujetos del grupo control tal y como se les informó al inicio de la investigación.

La presente investigación fue aprobada por el comité de bioética de la Universidad de Almería.

\section{Análisis estadístico}

Para comprobar la existencia de diferencias estadísticamente significativas entre las puntuaciones medias del grupo control y experimental en cada una de las fases del estudio para los componentes de la variable salud percibida, se empleó el estadístico de contraste no paramétrico $U$ de Man-Whitney para muestras independientes, ya que los datos no se ajustaban a la distribución normal de probabilidades

Para comprobar la existencia de diferencias estadísticamente significativas entre las puntaciones medias de las dimensiones de salud percibida en cada una de las fases del estudio en el grupo experimental y control, se empleó el estadístico de contraste no paramétrico de Wilcoxon para muestras relacionadas, ya que los datos no se ajustaban a la distribución normal de probabilidades.

Para valorar la magnitud del cambio experimentado tras la intervención aplicada tanto en la medida postest como en la medida de seguimiento en el grupo experimental, se empleó la $d$ de Cohen (Cohen, 1988) y el porcentaje de cambio entre las puntuaciones pretest-postest y pretest-seguimiento. Todos los análisis estadísticos fueron realizados con el paquete estadístico SPSS versión 15.0 .

\section{RESULTADOS}

En primer lugar, se procedió a calcular las medias y desviaciones típicas para la dimensiones de salud percibida correspondientes a los grupos control y experimental en cada una de las fases del estudio (ver Tablas 1 y 2).

Tabla 1. Medias y Desviaciones Típicas Pretest, Postest y Seguimiento en las Distintas Dimensiones de Salud Percibida en el Grupo Experimental

\begin{tabular}{lccccccc}
\hline & \multicolumn{2}{c}{ Pretest } & \multicolumn{2}{c}{ Postest } & \multicolumn{2}{c}{ Seguimiento } \\
\hline Variable & $M$ & $D T$ & $M$ & $D T$ & $M$ & $D T$ \\
\hline $\begin{array}{l}\text { Funcion. Físico } \\
-\cdots\end{array}$ & 14.09 & 6.34 & & 14.81 & 5.77 & 14.54 & 5.04 \\
Psy, Soc, \& Educ, 2010, Vol 2, No2 & & & & &
\end{tabular}




$\begin{array}{lllllll}\text { Rol físico } & 24.45 & 7.01 & 24.09 & 9.94 & 24.63 & 8.90 \\ \text { Dolor corporal } & 31.18 & 8.02 & 29.45 & 9.67 & 31.86 & 8.73 \\ \text { Salud general } & 29.36 & 9.42 & 30.78 & 11.2 & 31.45 & 8.76 \\ \text { Compon. Físico } & 21.27 & 5.34 & 21.72 & 6.87 & 21.45 & 7.38 \\ \text { Vitalidad } & 38.01 & 9.71 & 39.27 & 8.19 & 40.72 & 9.31 \\ \text { Funcion. Social } & 34.09 & 7.67 & 37.54 & 9.90 & 37.84 & 7.52 \\ \text { Rol emocional } & 42.91 & 9.01 & 46.63 & 8.75 & 46.90 & 9.68 \\ \text { Salud mental } & 35.09 & 7.36 & 38.45 & 9.92 & 39.27 & 8.70 \\ \text { Compon. Mental } & 41.54 & 10.2 & 45.63 & 9.97 & 46.27 & 8.49\end{array}$

Nota: Funcio. Fisico: Funcionamiento Físico; Compon. Físico: Componente Físico; Funcion. Social: Funcionamiento Social.

Tabla 2. Medias y Desviaciones Típicas Pretest, Postest y Seguimiento en las Distintas Dimensiones de Salud Percibida en el Grupo Control

\begin{tabular}{lcccccc}
\hline & \multicolumn{2}{c}{ Pretest } & \multicolumn{2}{c}{ Postest } & \multicolumn{2}{c}{ Seguimiento } \\
\hline Variable & $M$ & $D T$ & $M$ & $D T$ & $M$ & $D T$ \\
\hline Funcion. Físico & 12.79 & 4.11 & 13.48 & 6.08 & 11.87 & 5.38 \\
Rol físico & 22.93 & 6.25 & 20.82 & 7.38 & 22.51 & 9.15 \\
Dolor corporal & 33.02 & 9.82 & 34.18 & 8.72 & 32.27 & 7.94 \\
Salud general & 31.11 & 7.23 & 29.87 & 9.62 & 27.32 & 8.42 \\
Compon. Físico & 20.56 & 6.72 & 22.09 & 5.93 & 19.95 & 6.82 \\
Vitalidad & 40.52 & 10.3 & 38.61 & 8.87 & 41.03 & 10.1 \\
Funcion. Social & 32.91 & 6.41 & 34.06 & 7.12 & 32.68 & 8.23 \\
Rol emocional & 44.13 & 11.3 & 42.26 & 9.52 & 41.89 & 8.96 \\
Salud mental & 33.87 & 6.29 & 32.09 & 7.22 & 31.78 & 6.25 \\
Compon. Mental & 39.96 & 9.62 & 35.97 & 9.97 & 37.15 & 8.07 \\
& & & & & & \\
\hline
\end{tabular}

$\mathrm{Al}$ realizar la prueba $U$ de Man-Whitney para muestras independientes para las puntuaciones pretest, se observa como no hay diferencias significativas de partida entre las puntaciones medias del grupo control y experimental en las variables del estudio. Pero por el contrario, sí aparecieron diferencias estadísticamente significativas entre el grupo control y el experimental al realizar la diferencia de medias entre sus puntuaciones postest en las dimensiones componente mental, salud mental, dolor corporal, funcionamiento físico, salud general, rol emocional, y funcionamiento social. Al realizar la comparación de las puntuaciones medias en la medida de seguimiento entre el grupo control y el experimental, aparecieron diferencias significativas en las dimensiones salud mental, componente mental, rol emocional, y dolor general (ver Tabla 3). 
Tabla 3. Prueba U de Man-Whitney para Muestras Independientes de las Diferencias Pretest, Postest y Seguimiento entre el Grupo Control y Experimental en las Distintas Dimensiones de Salud Percibida

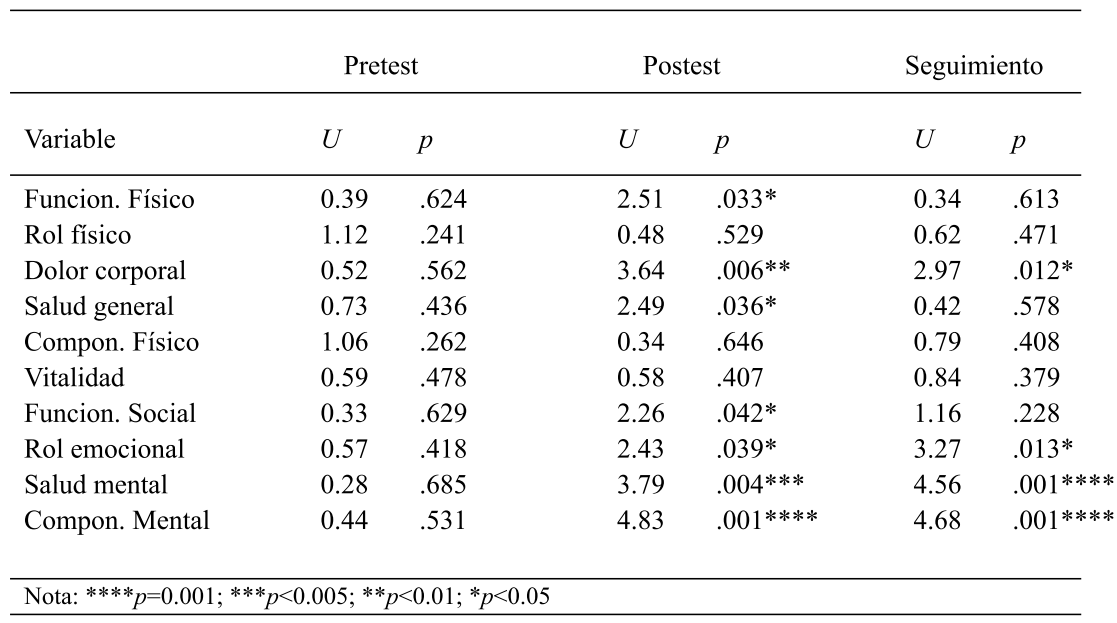

Al realizar la prueba de Wilcoxon para muestras relacionadas en el grupo experimental se obtuvo la existencia de diferencias significativas al comparar las puntuaciones pretest-postest en las dimensiones salud general, funcionamiento físico, dolor corporal, rol emocional, funcionamiento social, componente mental, y salud mental. Igualmente, al comparar las puntuaciones medias pretestseguimiento, también se obtuvieron diferencias significativas en las dimensiones salud general, funcionamiento social, rol emocional, salud mental, componente mental, y funcionamiento físico. Sin embargo, no aparecieron diferencias significativas al realizar el análisis de las puntuaciones postest-seguimiento en ninguna de las dimensiones de salud percibida en el grupo experimental (ver Tabla 4).

Tabla 4. Prueba de Wilcoxon para Muestras Relacionadas de las Diferencias Pretest-Postest, Pretest-Seguimiento y Postest-Seguimiento para las Distintas Dimensiones de Salud Percibida en el Grupo Experimental

\begin{tabular}{llllllll}
\hline & \multicolumn{2}{c}{ Pre-Post } & \multicolumn{2}{c}{ Pre-Seg. } & \multicolumn{2}{c}{ Post-Seg. } \\
\hline Variable & $W$ & $p$ & $W$ & $p$ & $W$ & $p$ \\
& & & & & & & \\
\hline Funcionamiento físico & 3.90 & $.003^{* *}$ & 2.45 & $.039^{*}$ & & 0.62 & .530 \\
Rol físico & 0.59 & .554 & 0.86 & .386 & 0.18 & .857 \\
Dolor corporal & 3.69 & $.004^{* *}$ & 0.42 & .670 & 0.14 & .886 \\
Componente físico & 1.09 & .273 & 0.36 & .713 & 0.63 & .525 \\
Salud general & 4.87 & $.001^{* * *}$ & 2.74 & $.029^{*}$ & 1.26 & .224
\end{tabular}




$\begin{array}{lllllll}\text { Funcionamiento social } & 2.77 & .026^{*} & 2.63 & .031^{*} & 0.18 & .852 \\ \text { Rol emocional } & 2.82 & .019^{*} & 2.54 & .034^{*} & 0.54 & .587 \\ \text { Salud mental } & 2.28 & .042^{*} & 2.68 & .029^{*} & 0.88 & .327 \\ \text { Componente mental } & 2.58 & .032^{*} & 2.35 & .037^{*} & 1.34 & .178\end{array}$

Nota: ${ }^{* * *} \mathrm{p}=.001 ; * * \mathrm{p}<.005 ;{ }^{*} \mathrm{p}<.05$.

Al realizar la prueba de Wilcoxon para muestras relacionadas para las dimensiones de la variable salud percibida en el grupo control, se obtuvieron diferencias significativas al realizar las comparaciones pretest-postest en la dimensión componente mental, obteniéndose una reducción de la puntuación media postest en comparación con la puntuación media pretest. También se obtuvieron diferencias significativas al realizar las comparaciones pretest-seguimiento en la dimensión salud general, con una reducción de la puntuación media de la medida de seguimiento en comparación con la puntuación media de la medida pretest. Finalmente, se obtuvieron diferencias estadísticamente significativas al realizar las comparaciones postest-seguimiento en la dimensión componente físico, con una reducción de la puntuación media de la medida de seguimiento en comparación con la puntuación media de la medida postest (ver Tabla 5).

Tabla 5. Prueba de Wilcoxon para Muestras Relacionadas de las Diferencias Pretest-Postest, Pretest-Seguimiento y Postest-Seguimiento en el Grupo Control para las Distintas Dimensiones de Salud Percibida

\begin{tabular}{|c|c|c|c|c|c|c|}
\hline \multirow[b]{2}{*}{ Variable } & \multicolumn{2}{|c|}{ Pre-Post } & \multicolumn{2}{|c|}{ Pre-Seg. } & \multicolumn{2}{|c|}{ Post-Seg. } \\
\hline & $W$ & $p$ & $W$ & $p$ & $W$ & $p$ \\
\hline Funcionamiento físico & 1.19 & .146 & 0.86 & .239 & 0.77 & .305 \\
\hline Rol físico & 0.81 & .242 & 1.14 & .189 & 0.13 & .809 \\
\hline Dolor corporal & 0.55 & .581 & 0.73 & .319 & 0.46 & .622 \\
\hline Componente físico & 0.49 & .614 & 0.43 & .622 & 2.26 & $.044^{*}$ \\
\hline Salud general & 0.65 & .458 & 2.46 & $.029 *$ & 0.53 & .584 \\
\hline Vitalidad & 0.23 & .792 & 0.71 & .446 & 0.48 & .611 \\
\hline Funcionamiento social & 0.59 & .572 & 0.49 & .607 & 0.58 & .552 \\
\hline Rol emocional & 0.88 & .226 & 1.02 & .219 & 0.64 & .461 \\
\hline Salud mental & 0.36 & .714 & 0.41 & .597 & 0.93 & .204 \\
\hline Componente mental & 2.39 & $.034 *$ & 0.61 & .469 & 0.31 & .742 \\
\hline
\end{tabular}

Con el objetivo de poder valorar la magnitud del cambio ocurrido tanto en la medida postest como en la medida de seguimiento, se empleó la $d$ de Cohen (Cohen 1988), donde valores superiores a 0.8 indican la presencia de cambios muy importantes, entre 0.5 y 0.8 importantes, por debajo de 0.5 medios, y menores de 0.2 indican cambios bajos. En la Tabla 6 se observa como las puntuaciones de la 
$d$ de Cohen para las medidas postest muestran la existencia de cambios de nivel medio en las dimensiones funcionamiento social, rol emocional, salud mental, y componente mental.

Por lo que respecta a la magnitud del cambio experimentado entre las puntuaciones pretest-seguimiento, se observa la existencia de cambios importantes en las dimensiones salud mental, y componente mental; mientras que aparecen cambios de nivel medio en las dimensiones funcionamiento social, rol emocional, vitalidad y salud general (ver Tabla 6).

Tabla 6. $d$ de Cohen y Porcentaje de Cambio Pretest-Postest y Pretest-Seguimiento en las Distintas Dimensiones de Salud Percibida en el Grupo Experimental

\begin{tabular}{lcccc}
\hline Variable & $d$ Postest & $d$ Segui. & \% Postest & \% Segui. \\
\hline Funcionamiento físico & 0.118 & 0.078 & 5.11 & 3.19 \\
Rol físico & 0.041 & 0.022 & 1.47 & 0.73 \\
Dolor corporal & 0.030 & 0.081 & 0.86 & 2.18 \\
Componente físico & 0.073 & 0.027 & 2.11 & 0.84 \\
Salud general & 0.137 & 0.230 & 4.83 & 6.64 \\
Vitalidad & 0.140 & 0.284 & 3.31 & 7.12 \\
Funcionamiento social & 0.389 & 0.494 & 10.1 & 11.1 \\
Rol emocional & 0.418 & 0.426 & 8.66 & 9.29 \\
Salud mental & 0.384 & 0.519 & 9.57 & 10.6 \\
Componente mental & 0.404 & 0.502 & 9.84 & 11.3 \\
& & & & \\
\hline
\end{tabular}

Finalmente, se calculó el porcentaje de cambio entre las puntuaciones pretestpostest y pretest-seguimiento. Respecto al porcentaje de cambio pretest-postest, en la Tabla 6 se observa como dicho porcentaje muestra unos incrementos entre el 8 y el $10 \%$ en las dimensiones funcionamiento social, rol emocional, salud mental, y componente mental; mientras que el porcentaje de cambio experimentado entre las puntuaciones pretest-seguimiento muestra unos incrementos entre el 9 y el $11 \%$ en esas mismas dimensiones.

\section{DISCUSIÓN}

Una vez analizados los resultados del presente estudio, podemos concluir que el programa de intervención en meditación mindfulness aplicado produjo mejoras significativas en varias dimensiones de salud percibida de las pacientes con fibromialgia del grupo experimental evaluadas mediante el cuestionario SF-36. El hecho de que las mejoras obtenidas se mantuviesen tres meses después de finalizada la intervención, suponen un apoyo al hecho de que mediante la práctica de las técnicas de conciencia plena la persona aprende una serie de habilidades que incorpora a su vida diaria y que pone en práctica en diversas situaciones. De 
este modo, las técnicas de conciencia plena suponen una alternativa tanto terapéutica como preventiva, aunque requieren de un compromiso activo por parte del paciente en su práctica regular (García-Campayo, 2008).

Estos resultados son acordes con los hallados por estudios que han demostrado la efectividad de las técnicas de meditación mindfulness para mejorar la sintomatología de pacientes con fibromialgia (Grossman et al., 2007; Sephton et al., 2007), así como con las conclusiones establecidas por García-Campayo et al. (2005) quienes comprobaron la eficacia de la intervención psicoeducativa de carácter grupal en el tratamiento de la fibromialgia.

No obstante las mejoras obtenidas en la presente investigación, afectan principalmente a las dimensiones social, emocional y mental de la salud percibida, y en menor medida a las dimensiones del área física relacionadas con la salud percibida. Esto puede deberse, a que el programa de intervención aplicado se dirige principalmente a mejorar el componente emocional de los participantes, y de este modo, estos resultados coincidirían con las asunciones de diversos investigadores (Craven, 1989; Delmonte, 1990; Walsh, 1999), quienes afirman la utilidad de las técnicas de meditación mindfulness en el desarrollo de habilidades relacionadas con la competencia emocional del individuo. En este sentido, la práctica de las técnicas de meditación mindfulness hace que la persona se pueda desvincular progresivamente de sus pensamientos y de sus procesos emocionales, convirtiéndose de esta manera en un mecanismo muy útil y efectivo de autorregulación emocional (Martín-Asuero y García de la Banda, 2007).

Por lo tanto, a la luz de los presentes resultados, se puede concluir que las técnicas de meditación mindfulness dada su demostrada eficacia, bajo coste, fácil aplicación, y sus prácticamente nulas contraindicaciones y efectos adversos, pueden ser incorporadas como parte de un tratamiento multidisciplinar para mejorar la sintomatología de personas diagnosticadas con fibromialgia.

No obstante, aunque los resultados del presente estudio han sido bastante alentadores para la mejora de diversas dimensiones de la salud percibida de pacientes con fibromialgia, hay que analizarlos con la debida cautela. En primer lugar, porque la muestra del estudio es relativamente pequeña, por lo que habría que confirmar dichos resultados con muestras más amplias. En segundo lugar, porque no se puede descartar la influencia de los factores inespecíficos sobre los resultados obtenidos, como la atención terapéutica, el apoyo social y la esperanza positiva. Es por ello que en futuras investigaciones habría que contar con un grupo de control placebo o con un grupo de comparación con intervención validada alternativa (manejo del estrés, entrenamiento en relajación, etc.), para garantizar de esta manera, que los resultaos obtenidos en el grupo experimental se deben al programa de intervención en meditación mindfulness. Además, deberían realizarse más medidas de seguimiento a largo plazo para comprobar si los resultados se mantienen a lo largo del tiempo. En tercer lugar, porque no se evaluaron características sociodemográficas de las participantes en el estudio como nivel de estudios, duración de la enfermedad, tratamiento farmacológico, estado civil, etc., por lo que dichas variables podrían estar incidiendo en los resultados obtenidos, 
de ahí la necesidad de controlar dichas variables en futuras investigaciones. Finalmente, sería recomendable realizar investigaciones cuyo objetivo fuese aislar qué componentes o elementos del programa son los responsables de los cambios y cuales no, con el propósito de mejorarlos y/o eliminarlos del mismo.

\section{Agradecimientos}

Queremos manifestar nuestro más profundo agradecimiento a la Asociación de Fibromialgia de Almería (AFIAL), por toda la ayuda prestada para la realización de esta investigación.

\section{REFERENCIAS}

Alonso, J., Prieto, L. y Antón J.M. (1995). La versión española del SF-36 Health Survey (Cuestionario de Salud SF-36): un instrumento para la medida de los resultados clínicos. Medicina Clínica, 104, 771- 776.

Alonso, J., Regidor, E., Barrio, G., Prieto, L., Rodríguez, C. y de la Fuente, L. (1998) Valores poblacionales de referencia de la versión española del Cuestionario de Salud SF-36. Medicina Clínica, 111, 410-416.

Ayuso-Mateos, J.L., Lasa, L. y Vázquez-Barquero JL. (1999). Validez interna y externa de la versión española del SF-36. Medicina Clínica, 113, 37-38.

Brown, G.K., Nicasio, P.M. y Wallston, K.A. (1989). Pain coping strategies and depression in rheumatoid arthritis. Journal of Consulting and Clinical Psychology, 57, 652-657.

Carricondo, M.I. (2009). Un pulso a la fibromialgia. Index de Enfermería, 18(2). Extraído el 13 de Julio de 2009 desde http://www.index-f.com/index-enfermeria/v18n2/6941.php.

Castro, M.E. (2007). La fibromialgia en el mejor momento de la vida. Index de Enfermería, 56. Extraído el 13 de julio de 2009 desde http://www.index-f. com/index-enfermeria/56/6434.php.

Cohen, J. (19888). Statistical power analysis for the behavioral sciences. Erlbaum: Hillsdale.

Collado, A., Aljiotos, J., Benito, P., Alegre, C., Romera, M., Sañuolo, I., et al. (2002). Documento de consenso sobre el diagnóstico y tratamiento de la fibromialgia en Cataluña. Medicina Clínica, 118, 745-749.

Craven, J.L. (1989). Meditation and psychotherapy. Canadian Journal of Psychiatry, 34, 648-653.

Delmonte, M.M. (1990). Meditation and change: Mindfulness versus repression. Australian Journal of Clinical Hypnotherapy and Hypnosis, 11(2), 57-63.

Durán, L., Gallegos, K., Salinas, G. y Martínez, H. (2004). Hacia una base normativa mexicana en la medición de calidad de vida relacionada con la salud mediante el Formato Corto 36. Salud Pública de México, 46(4), 306-315.

Franco, C. (2009). Meditación Fluir para serenar el cuerpo y la mente. Madrid: Bubok. 
García, A.M., Santibáñez, M. y Soriano, G. (2004). Utilización de un cuestionario de salud percibida (SF-36) en vigilancia de la salud de los trabajadores. Archivos de Prevención de Riesgos Labores, 7(3), 88-98.

García Campayo, J. (2008). La práctica del estar atento (mindfulness) en medicina. Impacto en pacientes y profesionales. Atención Primaria, 40(7), 363-366.

García-Campayo, J., Arnal, P., Marqués, H., Messeguer, E., Martínez, A., Navarro, C., et al. (2005). Intervención psicoeducativa en pacientes con fibromialgia en atención primaria: efectividad y diferencias entre terapia individual y grupal. Cuadernos de Medicina Psicosomática, 73, 32-41.

Garofalo, J.P. (2000). Perceived optimism and chronic pain. En R.J. Gatchel y J.N. Weisberg (Eds), Personality characteristics of patients with pain (pp. 61-88). Washington: Apa.

Grossman, P., Tiefenthaler-Gilmer, U., Raysz, A y Kiespes, U. (2007). Mindfulness training as an intervention for fibromyalgia: evidence of postintervention and 3-year follow-up benefits in web-being. Psychotherapy and Psychosomatics, 76, 226-233.

Hayes, S. C., Strosahl, K. D. y Wilson, K. G. (1999). Acceptance and Commitment Therapy: An experiential approach to behavior change. New York: Guilford Press.

Kabat-Zinn, J. (1990). Full catastrophe living: Using the wisdom of your body and mind to face stress, pain and illness. New York: Delacorte.

Linehan, M.M. (1993). Cognitive-behavioral treatment of borderline personality disorder. Nueva York: Guilford Press.

Martín-Asuero, A. y García de la Banda, G. (2007). Las ventajas de estar presente: desarrollando una conciencia plena para reducir el malestar psicológico. International Journal of Clinical and Health Psychology, 7(2), 369-384.

McHorney, C.A., Ware, J.E. y Raczeck, A.E. (1993). The MOS 36-Item Short Form Health Survey (SF-·36): II. Psychometric and clinical tests of validity in measuring physical and mental health constructs. Medical Care, 31, 247263.

Sephton, S.E., Salmon, P., Weissbecker, I., Ulmer, C., Floyd, A., Hoover, K. et al. (2007). Mindfulness meditation alleviates depressive symptoms in women with fibromyalgia: results of a randomized clinical trial. Arthritis and Rheumatism, 57, 77-85.

Soriano J y Monsalve V. (1999). Valoración, afrontamiento y emoción en pacientes con dolor crónico. Boletín de Psicología, 52, 43-64.

Turk, D.C. (1996). Effects of type of symptom onset on psychological distress and disability in fibromyalgia syndrome patients. Pain, 68, 423-430.

Vallejo, M.A. (2006). Mindfulness. Papeles del Psicólogo, 27(2), 92-99.

Vallejo, M.A. (2007). El Mindfulness y la "tercera generación de terapias psicológicas". Infocop, 33, 16-18.

Vilagut, G., Valderas, J.M., Ferrer, M., Garin, O., López-García, E., Alonso, J. (2008). Interpretación de los cuestionarios de salud SF-36 y SF-12 en España: componentes físico y mental. Medicina Clínica, 130(19), 726-735. 
Walsh, R. (1999). Assian contemplative disciplines: Common practices, clinical applications, and research findings. The Journal of Transpersonal Psychology, 31(2), 83-107.

Ware, J.E. y Sherbourne, C.D. (1992). The MOS 36-item short form health survey (SF-36)-I. Medical Care, 30, 473-483.

Wilson, K.G. y Luciano, M.C. (2002). Terapia de aceptación y compromiso. Un tratamiento conductual orientado a los valores. Madrid: Pirámide. 